\title{
Accessibility of Mobile Platforms
}

\author{
Alireza Darvishy \\ ZHAW Zurich University of Applied Sciences, Zurich, Switzerland \\ dvya@zhaw.ch
}

\begin{abstract}
This paper compares accessibility features of two popular platforms from a user perspective. The comparison is based on accessibility features for different kinds of disabilities such as vision, hearing or physically challenged users. A section on accessibility in mobile applications follows. According to a survey [1], the use of mobile platforms by people with disabilities is dramatically increasing. New accessibility features are introduced for each release of these platforms which makes them an affordable assistive technology.
\end{abstract}

Keywords: Accessibility, mobile devices, screen-readers, people with disabilities, assistive technologies, accessible apps.

\section{Introduction}

The use of mobile devices is growing rapidly, and the way we use mobile devices continues to evolve. Use of mobile devices for shopping, banking and other apps is to increasing and opening more opportunities to how we use mobile devices in daily life.

The advantages to people with disabilities are potentially more effective than those for the general population. Accessible mobile devices increase the ability of people with disabilities to shop, communicate, study and do other activities. Commercially available mobile devices can replace specialized hardware that many people with disabilities rely on for applications such as way-finding, reading and others.

The use of accessible mobile devices has recently increased dramatically, according to the last survey carried out by WebAim Screen Reader Survey 4, which reported that 71.8 percent of respondents indicated they use a screen reader on a mobile device, a 600 percent increase in mobile screen reader usage since the first survey was conducted just over 1 year ago.

There are a number of mobile devices on the market which offer accessibility features, including as Apple iOS, Google Android and BlackBerry. This paper considers the most popular mobile platforms, namely Apple iOS 7 and Google Android 4.4 $[2,3,4,5]$.

\section{Comparing Accessibility Features of iPhone and Android}

This section compares accessibility features of two popular mobile platforms: Apple iOS 7 and Google Android 4.4. The comparison is based on different senses such as 
vision, hearing, and physical and motor. The comparison is probably not complete and might be extended to other features.

Apple provides a strong base of accessibility features just out of the box. With the release of the iPhone 3GS in 2009, Apple developed the first mobile screen reader for touch-based devices. The VoiceOver [6] screen reader which is built into the Mac desktop platform was introduced into the iPhone. Apple provided a unique set of gestures to allow a user to nonvisually control the iPhone using VoiceOver. As a user's finger moves over or taps an element, the name of the element is spoken. Double tapping on the screen then activates the element.

Google, on the other hand, has an open source philosophy and this had led to adoption of a more "wild west" model of accessibility where developers are expected to create accessibility solutions rather than having them provided centrally.

\subsection{Vision}

\begin{tabular}{|c|c|c|}
\hline Feature(s) & iOS & Android \\
\hline Screen Reader & VoiceOver & TalkBack \\
\hline Zoom & Zoom & Magnification gestures \\
\hline Text magnification & $\begin{array}{l}\text { Large Text } \\
\text { - } 20 \mathrm{pt} \\
\text { - } 24 \mathrm{pt} \\
\text { - } 32 \mathrm{pt} \\
\text { - } 40 \mathrm{pt} \\
\text { - } 48 \mathrm{pt} \\
\text { - } 56 \mathrm{pt}\end{array}$ & $\begin{array}{l}\text { Font Size } \\
\text { - Tiny } \\
\text { - Small } \\
\text { - Normal } \\
\text { - Large } \\
\text { - Huge }\end{array}$ \\
\hline Bold Text & Bold Text & \\
\hline Colors & $\begin{array}{l}\text { Invert Colors } \\
\text { - White/Black } \\
\text { - Black/White }\end{array}$ & \\
\hline Contrast & Increase Contrast & \\
\hline Speak Selected Text & Speak Selection & \\
\hline Speech Rate & Speaking Rate & $\begin{array}{l}\text { Speech Rate } \\
\text { - Very slow } \\
\text { - Normal } \\
\text { - Fast } \\
\text { - Faster } \\
\text { - Very fast } \\
\text { - Rapid } \\
\text { - Very rapid } \\
\text { - Fastest }\end{array}$ \\
\hline
\end{tabular}




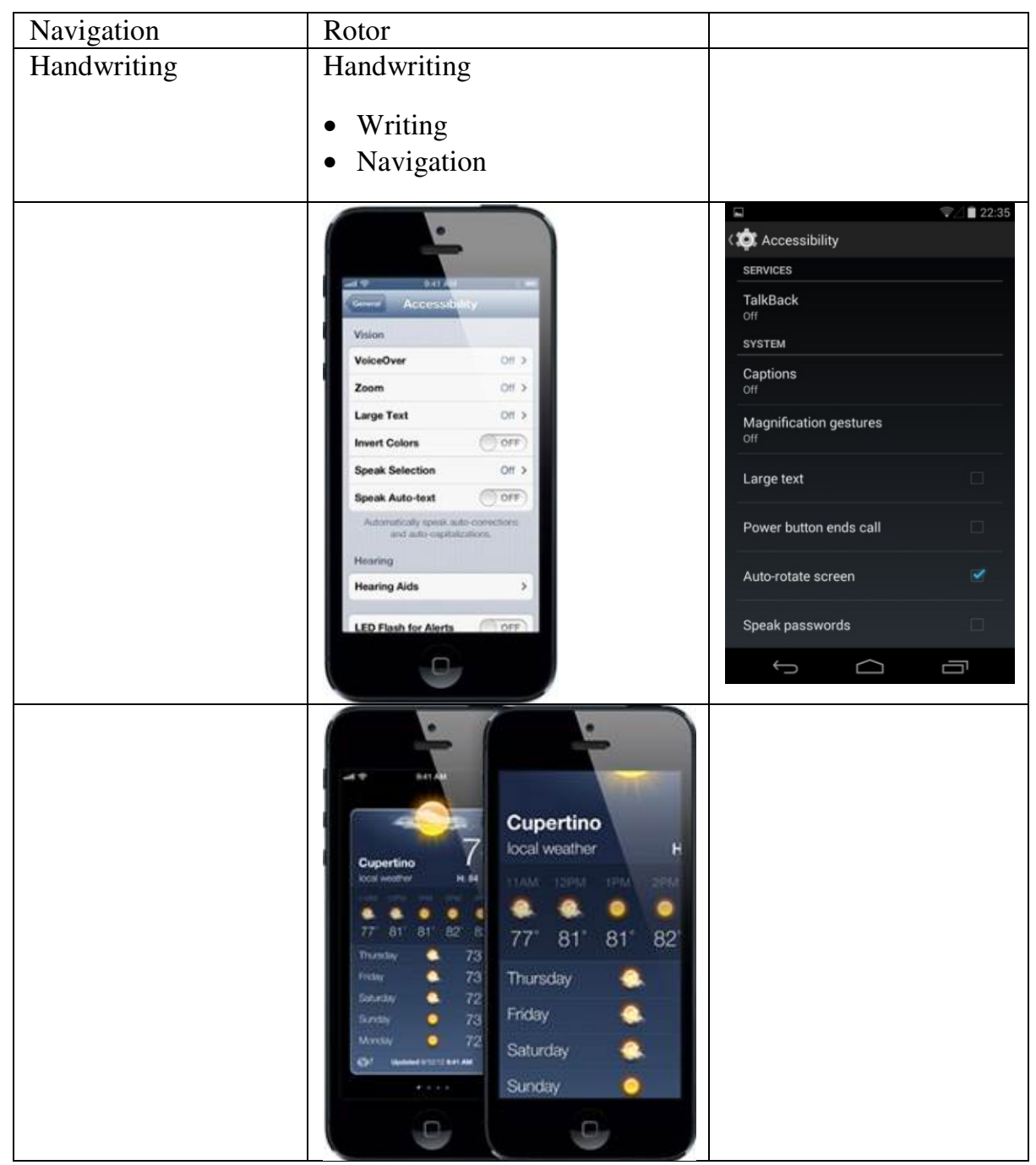




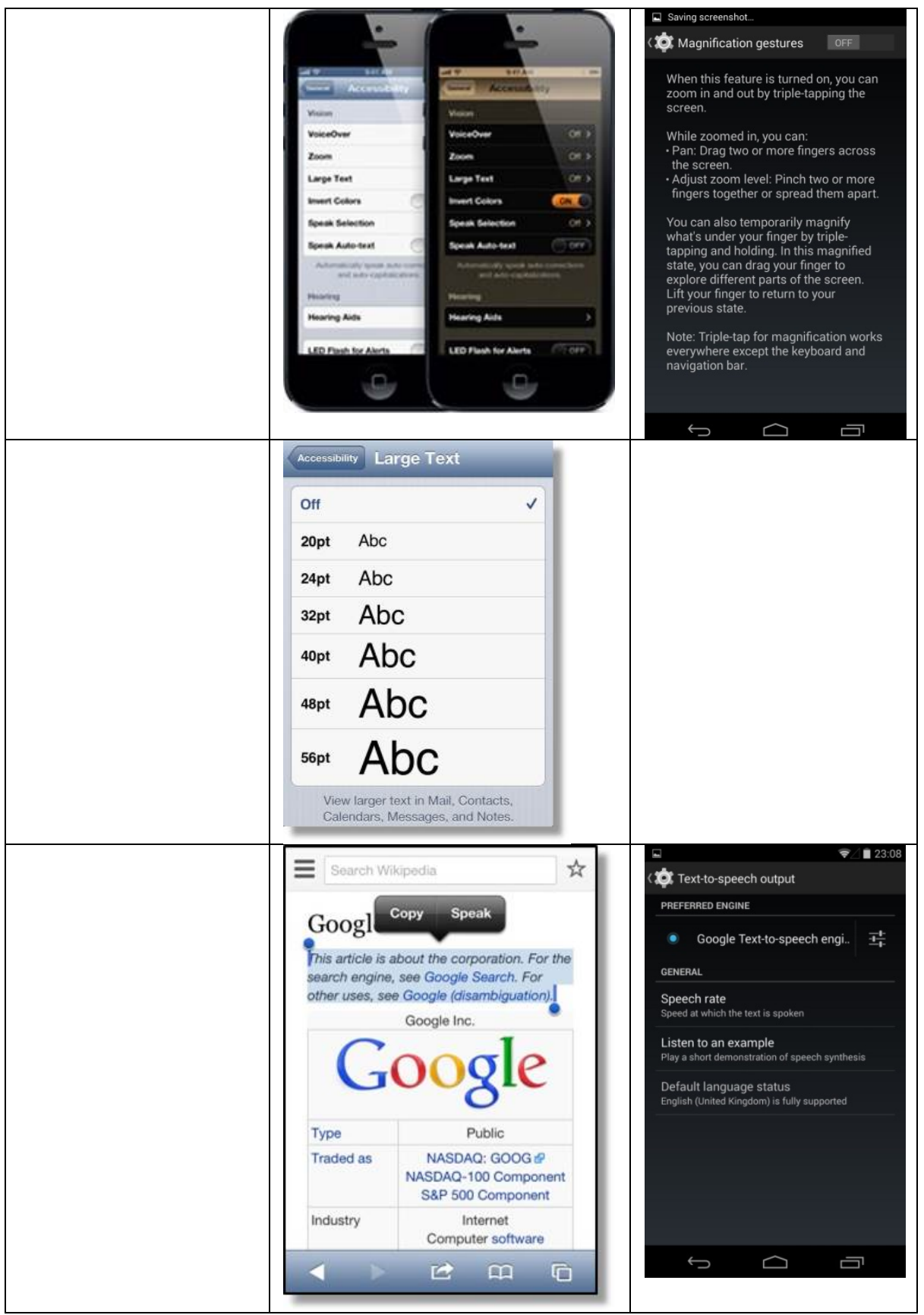




\section{$2.2 \quad$ Hearing}

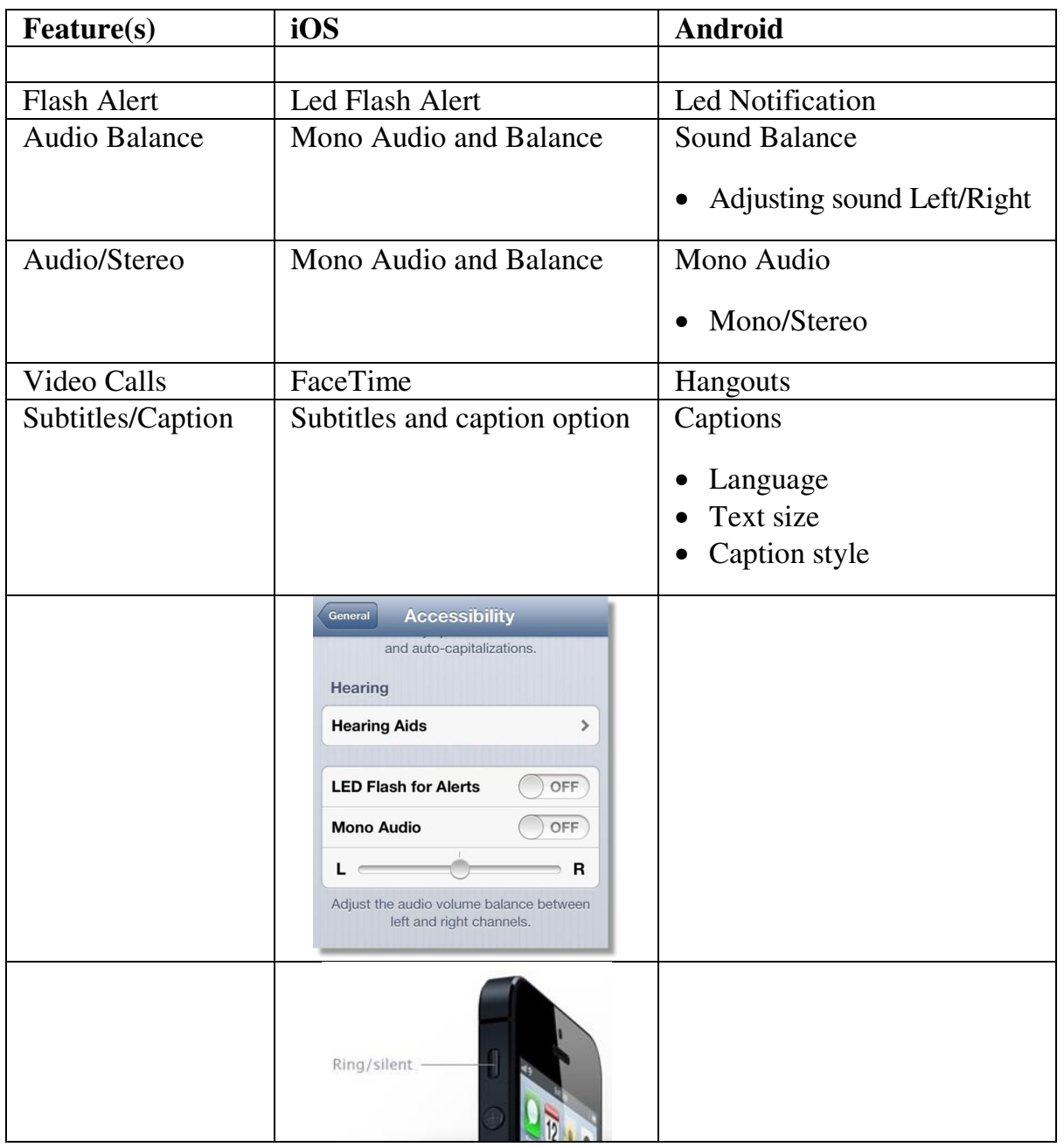

\subsection{Physical and Motor}

\begin{tabular}{|l|l|l|}
\hline Feature(s) & iOS & Android \\
\hline & & \\
\hline Touch Assistant & Assistive Touch & \\
\hline Incoming Calls & Incoming Calls & Answering/Ending Calls \\
& $\bullet$ Default & \\
& $\bullet$ Headset & \\
& $\bullet$ Speaker & \\
\hline
\end{tabular}




\begin{tabular}{|c|c|c|}
\hline $\begin{array}{l}\text { Accessibility click } \\
\text { speed }\end{array}$ & $\begin{array}{l}\text { Home-click Speed } \\
\text { - Default } \\
\text { - Slow } \\
\text { - Slowest }\end{array}$ & $\begin{array}{l}\text { Touch and hold delay } \\
\text { - Short } \\
\text { - Medium } \\
\text { - Long }\end{array}$ \\
\hline & 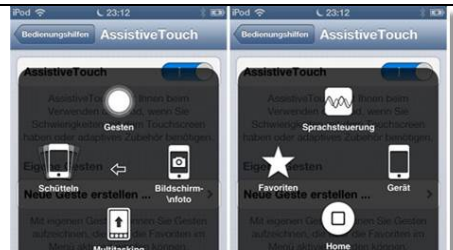 & \\
\hline
\end{tabular}

\subsection{Additional Features}

\begin{tabular}{|l|l|l|}
\hline Feature(s) & iOS & Android \\
\hline Speech Input & $\begin{array}{l}\text { SIRI } \\
\text { - Send messages } \\
\text { - Place phone calls } \\
\text { - Schedule meetings } \\
\text { - Set reminders } \\
\text { - Look up movie times } \\
\text { - Etc... }\end{array}$ & GoogleNow \\
\hline $\begin{array}{l}\text { Speak Password } \\
\text { Answering/ending }\end{array}$ & & $\begin{array}{l}\text { Speak Password: When ex- } \\
\text { plored by touch is enabled, } \\
\text { characters entered in password } \\
\text { fields read out by the device }\end{array}$ \\
\hline $\begin{array}{l}\text { Enable/Disable } \\
\text { Accessibility }\end{array}$ & & $\begin{array}{l}\text { Answering/ending calls: } \\
\text { - The home key answers calls } \\
\text { - Answer calls by tapping } \\
\text { Voice control } \\
\text { - The power key ends calls }\end{array}$ \\
\hline
\end{tabular}




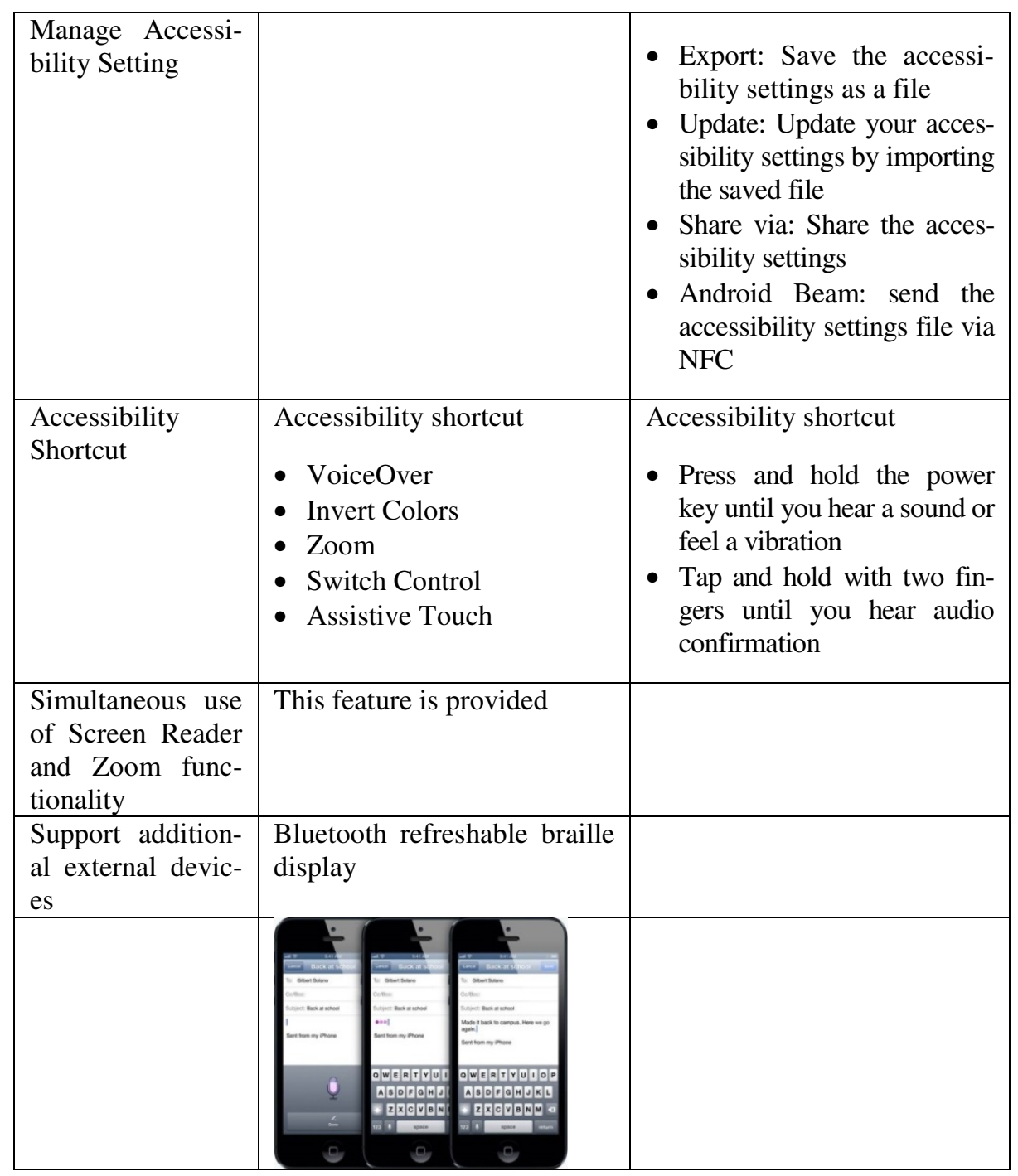

\subsection{Accessible Best Practices for Mobile Development}

Many fundamental accessible best practices will apply to mobile applications. Here are some examples:

- Text and images must meet relevant requirements for sufficient color contrast

- Screens must be laid out in an order that permits intuitive sequential navigation

- User interface controls must respond to multiple modalities of input

- User interface components must communicate correct information about the name, state, role and value of each component.

For a comprehensive description of the best practices see [7] for iOS and Android. 


\section{Conclusion}

This paper presented a comparison of accessibility features from two popular platforms. The presented features are not comprehensive, but it gives a good overview of currently available features. New trends and modalities such as force feedback [8] will provide good new accessibility features for users with disabilities in the future.

\section{References}

1. http://webaim.org/projects/screenreadersurvey/

2. http://support.apple.com/kb/HT5018?viewlocale=en_US

3. http://developer.android.com/design/patterns/ accessibility.html

4. http://mobileapps.blackberry.com/devicesoftware/ entry.do?code=bsr

5. http://docs.blackberry.com/en/smartphone_users/deliverables/2 $1882 /$ index. html ?name=Hearing+Aid+Compatibility+with+ BlackBerry+Smartphones+-+BlackBerry+Smartphones $5.0 \&$ language $=$ English\&userType=1\&category=BlackBerry+Smartphones\&subCategor y=Accessibility

6. https://www.webaccessibility.com/best_practices.php

7. http://eyes-free.googlecode.com/svn/trunk/documentation/ android_access/services.html 